Editorial

\title{
Schistosomus reflexus syndrome in dairy Egyptian cow (Under publication study)
}

\section{Editorial}

The exposure to a mixture of environmental pollution (air, water and feeding) has become a major cause of reproductive animal failure. They are major factors that adversely influence prenatal development. There is evidence to suggest that the developmental toxicological mechanisms of environmental pollution involve the generation of reactive oxygen species (ROS) and cellular oxidative damage. Overproduction of ROS induces oxidative stress, a state where increased ROS generation overwhelms antioxidant protection and subsequently leads to oxidative damage of cellular macromolecules. Major congenital anomalies are diagnosed in $2-4 \%$ of births. In this study (under publication), we review epidemiological studies that have specifically looked at congenital anomalies as a possible outcome of community exposure to environmental pollution. These include studies of drinking water contaminants (heavy metals and nitrates, chlorinated and aromatic solvents, and chlorination byproducts), residence near waste disposal sites and contaminated land, pesticide exposure in agricultural areas, air pollution and industrial pollution sources, food contamination.

From the economical points of view, a precautionary approach should be adopted at both community and individual level. In order to prevent congenital anomalies in animals, one must reduce exposure to potential environmental pollution before pregnancy is recognized.
Volume 2 Issue 4 - 2015

\author{
Gaber A Megahed \\ Theriogenology Department, Assiut University, Egypt
}

Correspondence: Gaber A Megahed, Theriogenology Department. Faculty of veterinary Medicine, Assiut University, Egypt, Tel 0100165 3725, Email gabermegahed@yahoo.com

Received: July 21, 2015 | Published: July 30, 2015

A pluriparus full term dairy cow in its 4th parity brought to clinical veterinary hospital, Faculty of veterinary Medicine, Assiut University, Egypt, with history of straining since last 6hours with no progression in parturition as well as it fails to make any progress towards delivery (Figure 1 \& Figure 2). Per vaginal examination revealed fully dilated cervix as well as it may detect a disoriented fetus and palpated only a three fetal's legs (Figure 3). To manage this dystocia, caesarean section was performed. Under local and high caudal epidural anaesthesia, caesarean-section was performed on the left flank region, adopting the standard operating procedure and the cow was positioned at standing condition (Figure $4 \&$ Figure 5). The Schistosoma reflex us fetus was extracted out (Figure $6 \&$ Figure 7).

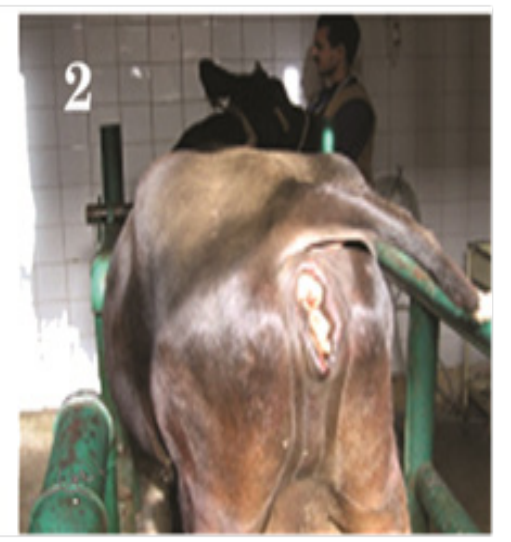

Figure I \& 2 History of straining since last 6hours with no progression in parturition as well as it fails to make any progress towards delivery.
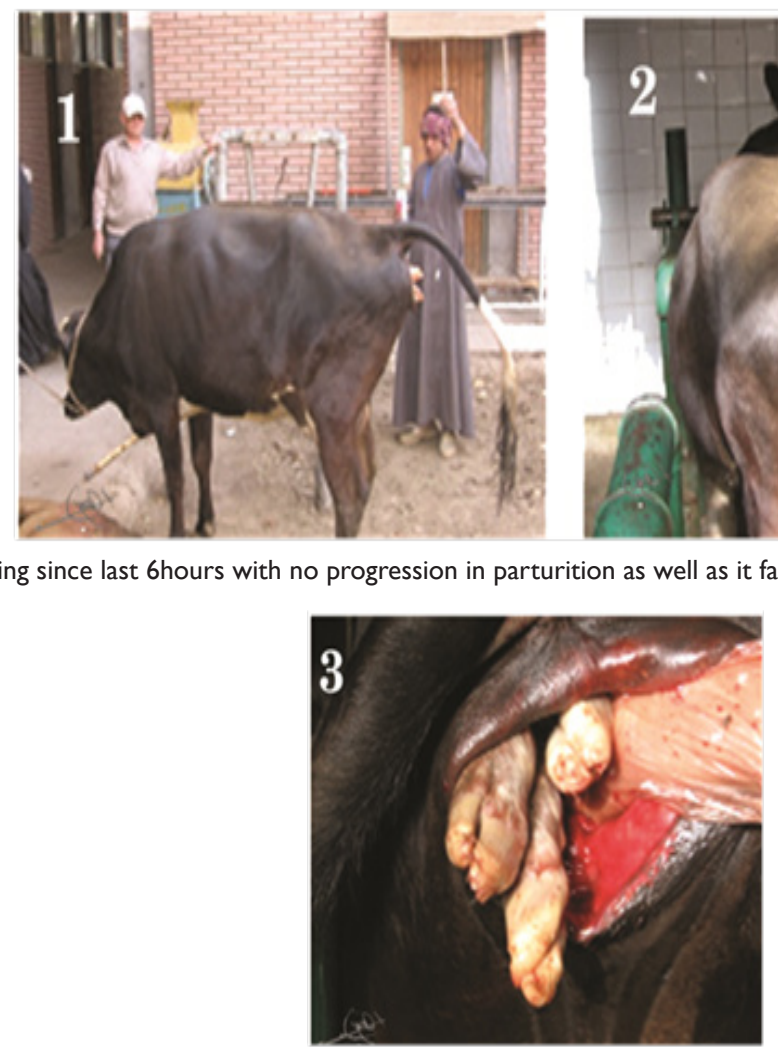

Figure 3 Per vaginal examination revealed fully dilated cervix as well as it may detect a disoriented fetus and palpated only a three fetal's legs. 

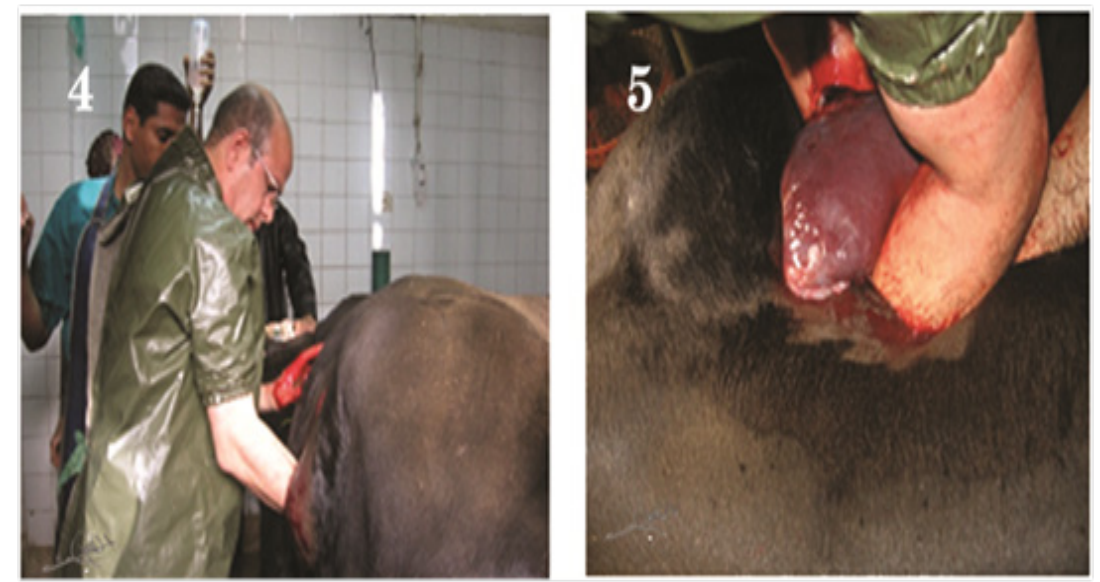

Figure 4 \& 5 Under local and high caudal epidural anaesthesia, caesarean-section was performed on the left flank region, adopting the standard operating procedure and the cow was positioned at standing condition.

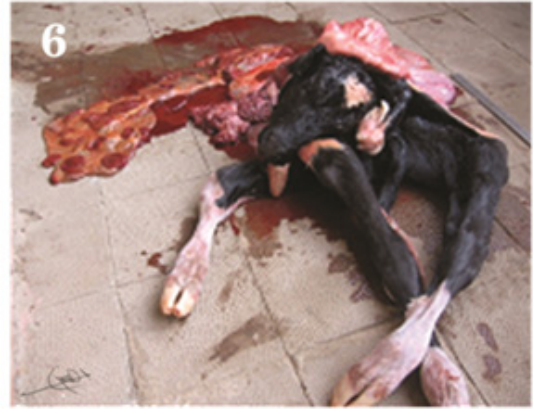

Figure 6 \& 7 The Schistosoma reflexus fetus was extracted out.

Schistosomus Reflexus (SR) is a birth defect resulting in the malformation of the entire body. Calves with this condition are commonly not able to pass through the birth canal, and must be retrieved by c-section or fetotomy. Its defining features include spinal inversion, exposure of the abdominal viscera because of a fissure of the ventral abdominal wall, limb ankylosis, positioning of the limbs adjacent to the skull and, lung and diaphragm hypoplasia. These abnormalities suggest SR occurs as early as the post-gastrulation embryo and involves the intermediate mesoderm. Preliminary analysis of associated cases suggests that SR has a genetic aetiology. ${ }^{1}$

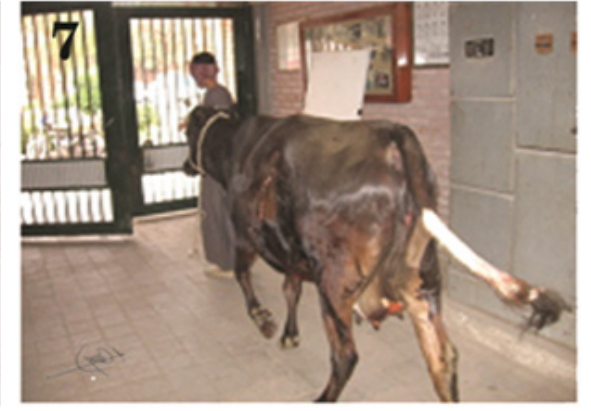

\section{Acknowledgements}

None.

\section{Conflict of interest}

Author declares that there is no conflict of interest.

\section{References}

1. Laughton KW, Fisher KR, Halina WG, et al. Schistosomus reflexus syndrome: a heritable defect in ruminants. Anat Histol Embryol. 2005;34(5):312-318. 